\title{
Using Collaborative Technologies for Chatbots' Creation, Development and Delivery
}

\author{
Luciano Gallegos ${ }^{1}$ \\ ${ }^{1}$ Universidade de Fortaleza (UNIFOR) \\ Research, Development and Innovation Department - Fortaleza - CE - Brazil \\ \{luciano.gallegos\}egmail.br
}

\begin{abstract}
The COVID-19 pandemic suddenly moved thousands for working remotely and since then had speeded up the use of collaborative technologies. In this industry report paper, we describe our 1-year experience using these collaborative technologies for the creation, development and delivery of chatbots, which are system used to conduct online conversation via text or speech, replacing a live human agent. This industry report experience gathered government professional, academic professors, researchers, graduate and undergraduate students, where 2 chatbots were successfully delivered.
\end{abstract}

\section{Introduction}

The dynamics in people's daily lives were strongly modified since the New Coronavirus (COVID-19) emergence, a respiratory disease discovered in China in late 2019. To avoid the collapse in the health care system, several cities in the world restricted, or forbid, people's mobility to diminish the COVID-19 spread among people [Lau et al. 2020]. In this industry report paper, we describe our 1-year experience in the city of Fortaleza, Ceará - Brazil, using collaborative technologies for the creation, development and delivery of chatbots (i.e. system used to conduct online conversation via text or text-to-speech, replacing a live human agent) for e-government initiatives, while team member were in quarantine or isolation during the COVID-19 pandemic in 2020.

\section{Collaboration and Tools}

Due to COVID-19 pandemic arrival in the Fortaleza area in February, 2020, we suddenly moved our offices home and the designing, development, testing and debugging, and deployment steps were then performed online and remotely by the team member. All these steps wouldn't be possible without Online Collaboration Tools - OCT, which are tools capable to support a group of two or more individuals to accomplish a common goal or objective [Lomas, Cyprien; Burke, Michael; Page, Carie L. 2008].

In our work, the following OCT were used: Google Workspace ${ }^{1}$ for meetings, agenda and application sharing, Whatsapp ${ }^{2}$ for exchanging short texts and voice messages, GitLab ${ }^{3}$ as a cloud DevOps platform, Docker ${ }^{4}$ as a standard unit of software that packages up code and all its dependencies (i.e. container), IBM Watson Assistant ${ }^{5}$ for

\footnotetext{
${ }^{1}$ https://workspace.google.com/

${ }^{2} \mathrm{https}: / /$ web.whatsapp.com/

${ }^{3}$ https://about.gitlab.com/

${ }^{4}$ https://www.docker.com/

${ }^{5}$ https://cloud.ibm.com/login
} 
chatbot's whole or small dependencies in dialogue systems, and MongoDB Atlas ${ }^{6}$ as a cloud database service for unstructured data. All these OCT are free, in cloud, and most team member were familiarized with them. Extra online training sections and tutorials were needed for leveraging team's OCT skill, which took around 50 days.

\section{Experience}

The chatbot project members were: one professor as team leader, two master researchers (one as project manager and designer, and the other as software developer), two undergraduate students as system developers, and two government professionals as stakeholders and system architecture designers. Most of these members knew each other before the COVID-19 outbreak, and project stakeholder was updated in monthly meetings about project status, online tests and discussions. Originally, just one chatbot was ordered by the Fortaleza city hall to help citizens' to better find online digital services while dialoguing by text.

The COVID-19 pandemic outbreak in Fortaleza encouraged team members to create another chatbot. Differently from the previous chatbot, which was focused on online digital services, this new chatbot called "Dr. Saúde" (or Dr. Health, in english) is specialized at screening COVID-19 symptoms and provide the nearby health care unit map to the citizen. Dr. Health was developed in 45 days and deployed in the beginning of April 2020, helping around 3,500 citizens per month since then. Later, the original chatbot project dedicated at helping citizens in searching online digital services was resumed, respecting the 1-year project deadline.

In both chatbot projects, milestones were respected and an online agenda for each team member was followed. Google Workspace and Whatsapp were easy to use, which helped online communication besides loosing projects' privacy. GitLab's and Docker's team knowledge in software engineering, management and integration were below expectations: extra training were necessary, and delayed the project in 2 months. IBM Watson Assistant is cloud service to build and deploy virtual assistants, but expensive once deployed: each dialogue between chatbot and a user is taxed. MongoDB Atlas stores chatbots' abilities as knowledge database for free, but is not stable, rendering chatbots' dialogues sometimes. The used of these OCT lead us to 3 important lessons: setup and maintain repositories and containers, weekly sprints, and constant communication are crucial for project success.

The projects were supported by the Fundação Cearence de Apoio ao Desenvolvimento Científico e Tecnológico - FUNCAP, the Coordination of Science, Technology and Innovation - CITINOVA, and the University of Fortaleza. This industry report paper author is very grateful to all these institutions.

\section{References}

Lau, H., Khosrawipour, V., Kocbach, P., Mikolajczyk, A., Schubert, J., Bania, J., and Khosrawipour, T. (2020). The positive impact of lockdown in Wuhan on containing the COVID-19 outbreak in China. Journal of Travel Medicine, 27(3).

Lomas, Cyprien; Burke, Michael; Page, Carie L. (2008). Collaboration tools. http: // www. ccti. colfinder. org. Online; accessed 11 January 2021.

\footnotetext{
${ }^{6}$ https://www.mongodb.com/
} 